\title{
Mental Health Gap - Intervention Guide training in Brazil: building integrated Primary Mental Health Care
}

\section{Helio Antonio Rocha* | Carolina Manso** | Kali Alves*** | Daniel Silveira Elia ${ }^{* * *}$ | Sandra Fortes ${ }^{\star * * * *}$ \\ *Psychiatrist, MSc, Assistant, Professor of Medical Psychology and Mental Health/School of Medical Sciences/LIPAPS Interdisciplinary Laboratory for Research on Primary Care/University of Rio de Janeiro State(UERJ), Brazil | **Psychologist, PhD, LIPAPS/UERJ/Brasil | ***Psychologist, MSc, LIPAPS/UERJ/Brasil | ${ }^{* * * * P s y c h o l o g i s t, ~ R i o ~ d e ~ J a n e i r o ~ S t a t e ~ H e a l t h ~}$ Secretary, Brazil | ${ }^{* \star \star \star *}$ Psychiatrist, PhD, Associate Professor of Medical Psychology and Mental Health in the School of Medical Sciences/ LIPAPS/UERJ , Brazil}

Objectives: To analyze trainings with Mental Health Gap Intervention Guide (MHGap-IG-version 1) for depression care in Brazil. To discuss the use of the MHGap-IG in organizing an Integrated Mental Health Network based on primary mental health care.

Background: Primary Mental Health Care is the most effective way of overcoming the mental health treatment gap. Building up providers' capacity within an integrated network is a key aspect.

Methods: The modules of Introduction, Depression, Suicide and Others from MHGap-IG Version 1.0 in Portuguese were used. Activities to develop integrated mental health care and teach the use of active methodologies were included. The training lasted two and a half days. Evaluation was held in the last hour of each meeting with an one hour activity using the questions: "so good; "so bad, so what" as ice-breakers.
Results: Trainings were held in 2017 in seven states, organized by PAHO-Brazil, LIPAPS/UERJ and State Health Secretaries. A total of 285 professionals participated, mainly mental health professionals(50\%) but also general practitioners $(13 \%)$, nurses $(22 \%)$ and managers, students or university professors. The biggest problems were the duration and the lack of discussion on alcohol and drugs abuse. Guaranteeing the continuity of the training, both through database materials or collaborative care, with joint consultations, were proposed.

Conclusion: The training was considered positive. However, continuous training through practical activities for the development of skills and help the integration between professionals of different categories were considered necessary. MHGap-IG version 2 has included supervision as a strategy for continuous education.

\section{Images of material used (printed manual cover and App images)}
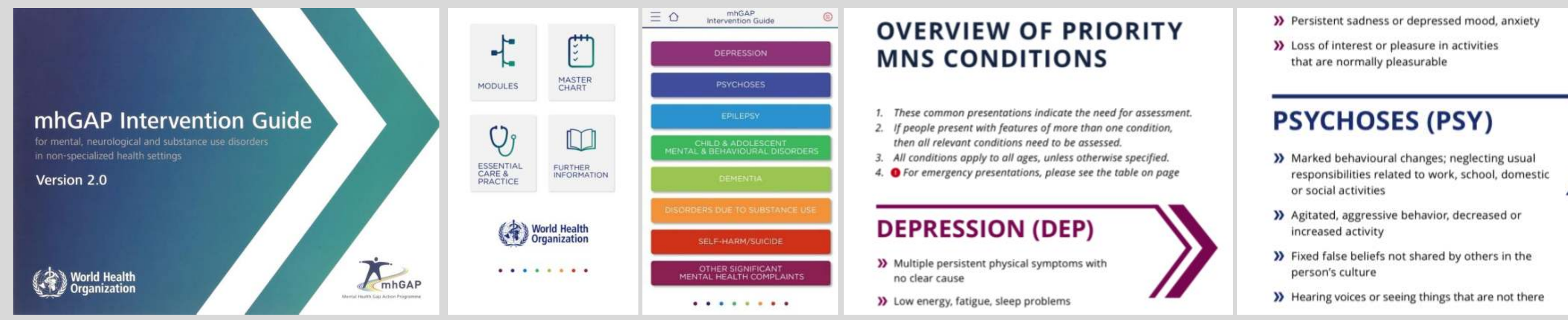

") Low energy, fatigue, sleep problems

https://www.who.int/mental health/publications/mhGAP intervention guide/en/ https://www.who.int/mental health/mhgap/mhGAP intervention guide 02/en/ 